\title{
ANALYSIS OF BLOCK-SOR ITERATION FOR THE THREE-DIMENSIONAL LAPLACIAN
}

\author{
WENJUN ZHENG ${ }^{1}$ and ZHIQIN ZHAO ${ }^{凶 2}$
}

(Received 1 November, 2007; revised 11 February, 2009)

\begin{abstract}
The successive over-relaxation (SOR) iteration method for solving linear systems of equations depends upon a relaxation parameter. A well-known theory for determining this parameter was given by Young for consistently ordered matrices. In this paper, for the three-dimensional Laplacian, we introduce several compact difference schemes and analyse the block-SOR method for the resulting linear systems. Their optimum relaxation parameters are given for the first time. Analysis shows that the value of the optimum relaxation parameter of block-SOR iteration is very sensitive for compact stencils when solving the three-dimensional Laplacian. This paper provides a theoretical solution for determining the optimum relaxation parameter in real applications.

2000 Mathematics subject classification: primary 65F10.

Keywords and phrases: compact stencil, block-SOR iteration, optimum relaxation parameter, three-dimensional Laplacian.
\end{abstract}

\section{Introduction}

The successive over-relaxation (SOR) iteration method [3, 6] is very popular for solving large sparse systems of linear algebraic equations. Convergence of the method is greatly affected by the choice of relaxation parameter $\omega$. A theory for determining $\omega$ for consistently ordered matrices was given by Young in [7]. That is, if $A$ is a consistently ordered matrix, then the eigenvalues $\mu$ and $\lambda$ of the Jacobi and SOR iteration matrices satisfy the well-known relationship $(\lambda+\omega-1)^{2}=\omega^{2} \mu^{2} \lambda$. Based on this relationship, if each $\mu$ is real, then the optimum relaxation parameter for SOR iteration can be expressed by the spectral radius of the Jacobi iteration matrix. This theory is applied in the paper for determining the optimum relaxation parameters.

For the model problem, (a system of equations arising from a finite difference approximation of the two-dimensional Laplace's equation or Poisson's equation on

\footnotetext{
${ }^{1}$ School of Electronic Engineering, University of Electronic Science and Technology of China, Chengdu, Sichuan, PR China; e-mail: janal_zheng@uestc.edu.cn.

${ }^{2}$ School of Electronic Engineering, University of Electronic Science and Technology of China, Chengdu, Sichuan, PR China; e-mail: zqzhao@uestc.edu.cn.

(C) Australian Mathematical Society 2009, Serial-fee code 1446-1811/2009 \$16.00
} 
a rectangle with Dirichlet boundary conditions) SOR iteration for a five-point stencil was analysed by Young in [8] and by Xie and Adams in [2]. In [1], optimum relaxation parameters for point-SOR and line-SOR iterations for a nine-point stencil [5]. In [4], by introducing Fourier analysis, the optimal $\omega$ and corresponding convergence rate were obtained rigorously. However, these analyses of the problem are in two dimensions. In this paper, we consider the problem in three dimensions and introduce several compact difference schemes correspondingly. By separating variables and solving difference equations, we give the optimum relaxation parameters of blockSOR iteration with natural row-wise ordering for the compact stencils for the threedimensional Laplacian.

This paper is organized as follows: in Section 2, we introduce several compact difference schemes for the three-dimensional Laplacian. Block-SOR iteration methods are briefly reviewed in order to give better understandings for the following sections. In Section 3, by deriving the spectral radius of a block-Jacobi iteration matrix, we present the optimum relaxation parameter of block-SOR iteration for the compact stencil for the three-dimensional Laplacian. Some conclusions are drawn in Section 4.

\section{Preliminaries}

2.1. The compact difference scheme approximation to the three-dimensional Laplacian The Laplacian in three dimensions for $u \equiv u(x, y, z)$ is abbreviated as $\Delta u$, where

$$
\Delta u=\frac{\partial^{2} u}{\partial x^{2}}+\frac{\partial^{2} u}{\partial y^{2}}+\frac{\partial^{2} u}{\partial z^{2}} .
$$

Assume the function $u(x, y, z)$ is defined on $\Omega=\{(x, y, z) \mid 0 \leq x, y, z \leq 1\}$ (a unit cubic region). Superimpose on the region $\Omega$ a cubic network with mesh size $h=$ $1 /(N+1)$ in the $x, y$ and $z$ directions. The region $\Omega$ is replaced by a set of grid points denoted by $\left(x_{j}, y_{k}, z_{m}\right)$ where $x_{j}=j \cdot h$ on the $X$-axis, $y_{k}=k \cdot h$ on the $Y$-axis and $z_{m}=m \cdot h$ on the $Z$-axis, $j, k, m=0,1,2, \ldots, N+1$. Denote the set of interior grid points by $\Omega_{h}=\left\{\left(x_{j}, y_{k}, z_{m}\right) \mid j, k, m=1,2, \ldots, N\right\}$. Let $P\left(x_{j}, y_{k}, z_{m}\right)$ be an arbitrary element of $\Omega_{h}$ : its neighbouring grid points denoted as 1-26 are shown in Figure 1.

Define the unit displacement operator as $I u\left(x_{j}, y_{k}, z_{m}\right)=u\left(x_{j}, y_{k}, z_{m}\right)$ and the displacement operators on $X, Y, Z$-axis directions as

$$
\begin{gathered}
\Gamma_{x} u\left(x_{j}, y_{k}, z_{m}\right)=u\left(x_{j+1}, y_{k}, z_{m}\right), \quad \Gamma_{y} u\left(x_{j}, y_{k}, z_{m}\right)=u\left(x_{j}, y_{k+1}, z_{m}\right), \\
\Gamma_{z} u\left(x_{j}, y_{k}, z_{m}\right)=u\left(x_{j}, y_{k}, z_{m+1}\right) .
\end{gathered}
$$

Evidently, the displacement operators above are invertible. For example, $\Gamma_{x}^{-1} u\left(x_{j}, y_{k}, z_{m}\right)=u\left(x_{j-1}, y_{k}, z_{m}\right)$. The products of the displacement operators and their inverses build multi-step displacement operators in which multiplication is commutative. According to their operation steps to $I u\left(x_{j}, y_{k}, z_{m}\right)$ we classify them into three groups and define three corresponding symmetry sums of operators 


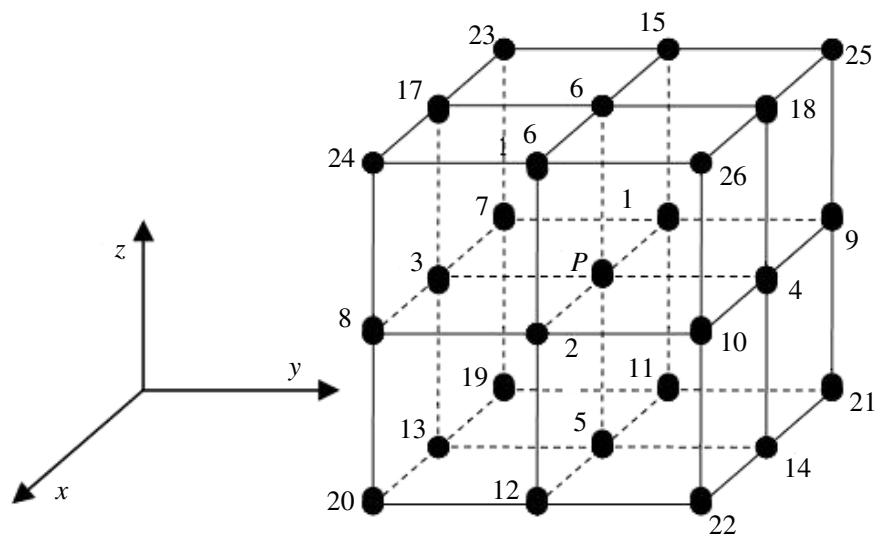

FIGURE 1 . The 27 grid points in a reference cube.

as follows:

$$
\begin{aligned}
T_{1}= & \Gamma_{x}+\Gamma_{x}^{-1}+\Gamma_{y}+\Gamma_{y}^{-1}+\Gamma_{z}+\Gamma_{z}^{-1} \\
T_{2}= & \Gamma_{x} \Gamma_{y}+\Gamma_{x} \Gamma_{z}+\Gamma_{y} \Gamma_{z}+\Gamma_{x}^{-1} \Gamma_{y}+\Gamma_{x}^{-1} \Gamma_{z}+\Gamma_{x} \Gamma_{y}^{-1}+\Gamma_{x} \Gamma_{z}^{-1} \\
& +\Gamma_{y} \Gamma_{z}^{-1}+\Gamma_{y}^{-1} \Gamma_{z}+\Gamma_{x}^{-1} \Gamma_{y}^{-1}+\Gamma_{x}^{-1} \Gamma_{z}^{-1}+\Gamma_{y}^{-1} \Gamma_{z}^{-1}, \\
T_{3}= & \Gamma_{x} \Gamma_{y} \Gamma_{z}+\Gamma_{x}^{-1} \Gamma_{y}^{-1} \Gamma_{z}^{-1}+\Gamma_{x}^{-1} \Gamma_{y} \Gamma_{z}+\Gamma_{x} \Gamma_{y}^{-1} \Gamma_{z}^{-1}+\Gamma_{x}^{-1} \Gamma_{y}^{-1} \Gamma_{z} \\
& +\Gamma_{x} \Gamma_{y} \Gamma_{z}^{-1}+\Gamma_{x}^{-1} \Gamma_{y} \Gamma_{z}^{-1}+\Gamma_{x} \Gamma_{y}^{-1} \Gamma_{z} .
\end{aligned}
$$

THEOREM 2.1. Suppose $u(x, y, z)$ is continuously differentiable for any order on $\Omega$. Let $T=\alpha T_{1}+\beta T_{2}+\gamma T_{3}$, where $\alpha, \beta$ and $\gamma$ are three nonnegative constants. Define the three differential operators

$$
\varphi=h \frac{\partial}{\partial x}, \quad \sigma=h \frac{\partial}{\partial y} \quad \text { and } \quad \tau=h \frac{\partial}{\partial z} .
$$

Then we have

$$
\begin{aligned}
T= & (6 \alpha+12 \beta+8 \gamma) I+(\alpha+4 \beta+4 \gamma) h^{2} \Delta+\frac{1}{12}(\alpha+4 \beta+4 \gamma) h^{4} \Delta^{2} \\
& +\frac{1}{6}(2 \beta+8 \gamma-\alpha)\left(\varphi^{2} \sigma^{2}+\varphi^{2} \tau^{2}+\sigma^{2} \tau^{2}\right)+O\left(h^{6}\right) .
\end{aligned}
$$

PROOF. By use of truncated Taylor series expansions of $u\left(x_{j+\delta_{1} h}, y_{k+\delta_{2} h}, z_{m+\delta_{3} h}\right)$ on $P\left(x_{j}, y_{k}, z_{m}\right)$ (with $\left.\delta_{1}, \delta_{2}, \delta_{3}=0, \pm 1\right),(2.1)$ can be transformed into

$$
\begin{aligned}
T_{1}= & 6 I+\left(\varphi^{2}+\sigma^{2}+\tau^{2}\right)+\frac{1}{12}\left(\varphi^{4}+\sigma^{4}+\tau^{4}\right)+O\left(h^{6}\right), \\
T_{2}= & 12 I+4\left(\varphi^{2}+\sigma^{2}+\tau^{2}\right)+\frac{1}{3}\left(\varphi^{4}+\sigma^{4}+\tau^{4}\right) \\
& +\left(\varphi^{2} \sigma^{2}+\varphi^{2} \tau^{2}+\sigma^{2} \tau^{2}\right)+O\left(h^{6}\right), \\
T_{3}= & 8 I+4\left(\varphi^{2}+\sigma^{2}+\tau^{2}\right)+\frac{1}{3}\left(\varphi^{4}+\sigma^{4}+\tau^{4}\right) \\
& +2\left(\varphi^{2} \sigma^{2}+\varphi^{2} \tau^{2}+\sigma^{2} \tau^{2}\right)+O\left(h^{6}\right) .
\end{aligned}
$$


Adding these after multiplying by $\alpha, \beta$ and $\gamma$, respectively gives

$$
\begin{aligned}
T= & (6 \alpha+12 \beta+8 \gamma) I+(\alpha+4 \beta+4 \gamma)\left[\left(\varphi^{2}+\sigma^{2}+\tau^{2}\right)+\frac{1}{12}\left(\varphi^{4}+\sigma^{4}+\tau^{4}\right)\right] \\
& +(\beta+2 \gamma)\left(\varphi^{2} \sigma^{2}+\varphi^{2} \tau^{2}+\sigma^{2} \tau^{2}\right)+O\left(h^{6}\right) .
\end{aligned}
$$

The Laplacian can also be expressed through $\varphi, \sigma$ and $\tau$ as

$$
\begin{gathered}
h^{2} \Delta=\varphi^{2}+\sigma^{2}+\tau^{2}, \\
h^{4} \Delta^{2}=\left(\varphi^{2}+\sigma^{2}+\tau^{2}\right)^{2}=\left(\varphi^{4}+\sigma^{4}+\tau^{4}\right)+2\left(\varphi^{2} \sigma^{2}+\varphi^{2} \tau^{2}+\sigma^{2} \tau^{2}\right) .
\end{gathered}
$$

By (2.3) and (2.4), (2.2) is obtained. Therefore, Theorem 2.1 is proved.

In fact, (2.2) gives an approximate operator $T$ for the Laplacian. Commonly, the operator $T$ has second-order precision approximating to the Laplacian. However, if we let the right-hand side of (2.2) be a linear combination of $I, h^{2} \Delta$ and $h^{4} \Delta^{2}$ (which implies the required condition of $\alpha=2 \beta+8 \gamma$ ), $T$ becomes a fourth-order precision operator approximating the Laplacian. Let the approximation operator $T$ operate on the function $u(x, y, z)$ to yield the compact difference schemes for the three-dimensional Laplacian. We give four traditional difference schemes.

(1) Seven-point difference scheme: $\alpha=1, \beta=\gamma=0$,

$$
\frac{T_{1} u-6 u}{h^{2}}=\Delta u+O\left(h^{2}\right) .
$$

(2) 15-point difference scheme: $\alpha=8, \beta=0, \gamma=1$,

$$
\frac{8 T_{1} u+T_{3} u-56 u}{12 h^{2}}=\Delta u+\frac{1}{12} h^{2} \Delta^{2} u+O\left(h^{4}\right) .
$$

(3) 19-point difference scheme: $\alpha=2, \beta=1, \gamma=0$,

$$
\frac{2 T_{1} u+T_{2} u-24 u}{6 h^{2}}=\Delta u+\frac{1}{12} h^{2} \Delta^{2} u+O\left(h^{4}\right) .
$$

(4) 27-point difference scheme: $\alpha=16, \beta=4, \gamma=1$,

$$
\frac{16 T_{1} u+4 T_{2} u+T_{3} u-152 u}{36 h^{2}}=\Delta u+\frac{1}{12} h^{2} \Delta^{2} u+O\left(h^{4}\right) .
$$

Let $u_{j, k, m}(j, k, m=0,1,2, \ldots, N+1)$ denote the approximate value of $u\left(x_{j}, y_{k}, z_{m}\right)$. Set

$$
X=\left(u_{1,1,1}, u_{2,1,1}, \ldots, u_{N, 1,1}, u_{1,2,1}, \ldots, u_{N, N, 1}, \ldots, u_{N, N, N}\right)^{T} .
$$

The compact difference schemes (2.5)-(2.8) with a boundary condition define a discrete analogue of the three-dimensional Laplacian. Moving the known values to the right-hand side, we obtain a linear system

$$
A X=b,
$$

where $A$ is an $N^{3}$ matrix, $X$ and $b$ are $N^{3}$-dimensional column vectors. 
The sparsity patterns of the coefficient matrix $A$ are illustrated by Figure 2 . We see that $A$ holds the tri-diagonal block form as

$$
A=\left(\begin{array}{ccccc}
B & -C & & & \\
-C & B & -C & & \\
& \ddots & \ddots & \ddots & \\
& & -C & B & -C \\
& & & -C & B
\end{array}\right)
$$

For convenience, we denote $A$ as Tridiag $(-C, B,-C)$. Thereby, the subblocks $B$ and $C$ of order $N^{2}$ can be presented as $B=\operatorname{Tridiag}(-F, D,-F)$ and $C=$ $\operatorname{Tridiag}(H, F, H)$, where $D=\operatorname{Tridiag}(-\alpha, d,-\alpha), F=\operatorname{Tridiag}(\beta, \alpha, \beta)$ and $H=$ $\operatorname{Tridiag}(\gamma, \beta, \gamma)$ are three subblocks of order $N, d=6 \alpha+12 \beta+8 \gamma$.

2.2. The block-SOR iteration method Suppose a linear system of order $n$ is partitioned into the block form

$$
\left(\begin{array}{cccc}
A_{11} & A_{12} & \cdots & A_{1 p} \\
A_{21} & A_{22} & \cdots & A_{2 p} \\
\vdots & \vdots & \ddots & \vdots \\
A_{p 1} & A_{p 2} & \cdots & A_{p p}
\end{array}\right)\left(\begin{array}{c}
X_{1} \\
X_{2} \\
\vdots \\
X_{p}
\end{array}\right)=\left(\begin{array}{c}
b_{1} \\
b_{2} \\
\vdots \\
b_{p}
\end{array}\right)
$$

where $A_{i, i}$ of order $n_{i}$ are nonsingular matrices, $X_{i}$ and $b_{i}$ are the $n_{i}$-dimensional sub-vectors, $i=1,2, \ldots, p, n_{1}+n_{2}+\cdots n_{q}=n$. Note $D=A+L+R$, where

$$
L=-\left(\begin{array}{cccc}
0 & & & \\
A_{21} & 0 & & \\
\vdots & \ddots & \ddots & \\
A_{p 1} & \cdots & A_{p, p-1} & 0
\end{array}\right), \quad R=-\left(\begin{array}{cccc}
0 & A_{12} & \cdots & A_{1 p} \\
& 0 & \ddots & \vdots \\
& & \ddots & A_{p-1, p} \\
& & & 0
\end{array}\right)
$$

Then, the block-Jacobi iteration can be written as $X^{(k+1)}=B_{J} X^{(k)}+f_{J}$, where $B_{J}=D^{-1}(L+R)$ is the Jacobi iteration matrix, $f_{J}=D^{-1} b$.

The block-SOR iteration can be written as $X^{(k+1)}=\mathcal{L}_{\omega} X^{(k)}+f_{\omega}$, where $\mathcal{L}_{\omega}=$ $(D-\omega L)^{-1}[(1-\omega) D+\omega R]$ is the SOR iteration matrix and $f_{\omega}=\omega(D-\omega L)^{-1} b$.

Definition 2.2. Given a matrix $A=\left(a_{i, j}\right)_{n \times n}$, the integers $i$ and $j$ are associated with respect to $A$ if $a_{i, j} \neq 0$ or $a_{j, i} \neq 0$.

Definition 2.3 ([8]). Suppose $A$ of order $n$ is partitioned into the form (2.11). Matrix $A$ is consistently ordered if for some $t$ there exist $t$ disjoint subsets $S_{1}, S_{2}, \ldots, S_{t}$ of $W=\{1,2, \ldots, p\}$ such that $\sum_{k=1}^{t} S_{k}=W$ and such that if $i$ and $j$ are associated, then $j \in S_{k+1}$ if $j>i$ and $j \in S_{k-1}$ if $j<i$, where $S_{k}$ is the subset containing $i$. 


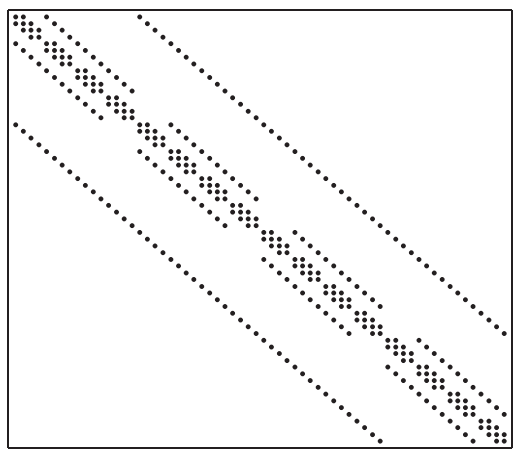

7-point stencil

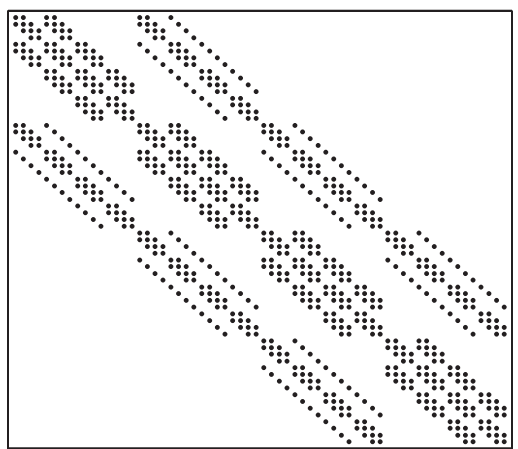

19-point stencil

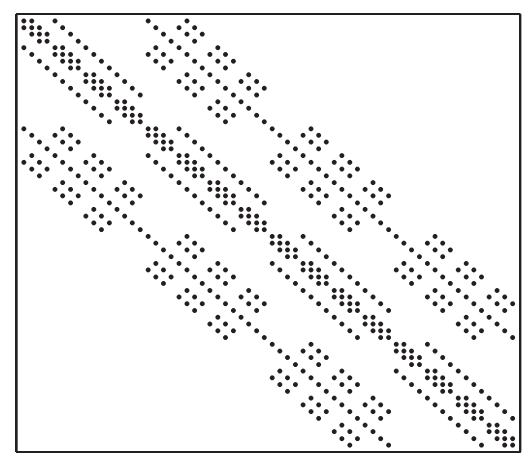

15 -point stencil

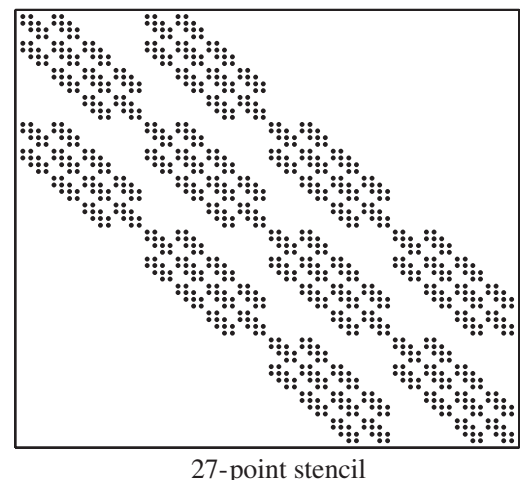

FIGURE 2. The sparsity patterns of $A$ corresponding to the four compact stencils with $h=1 / 5$ in natural row-wise ordering.

THEOREM 2.4 ([7]). Suppose the coefficient matrix A of (2.11) is consistently ordered, with nonzero diagonal elements. Furthermore, assume all the eigenvalues of the Jacobi iteration matrix are real. Then the SOR method converges if and only if

$$
0<\omega<2 \text { and } \rho\left(\mathcal{L}_{\omega}\right)<1,
$$

where $\rho(\cdot)$ denotes spectral radius.

THEOREM 2.5 ([7]). Let $\omega_{b}$ denote the optimum relaxation parameter for SOR iteration. For the condition of Theorem 2.4, $\omega_{b}$ and the spectral radius of Jacobi iteration matrix $\rho\left(B_{J}\right)$ satisfy the relationship

$$
\omega_{b}=\frac{2}{1+\sqrt{1-\rho^{2}\left(B_{J}\right)}} .
$$

\section{Determining the optimum relaxation parameters}

If we confirm that $A$ is consistently ordered in the block form of (2.10), using Theorems 2.4 and 2.5, we can easily obtain the optimum relaxation parameter $\omega_{b}$ for 
the block-SOR iteration if the spectral radius of $B_{J}$ is determined. In this section, two useful lemmas are proposed and proved. Then the optimum relaxation parameters for block-SOR iteration are determined.

LEMMA 3.1. The coefficient matrix A of linear system (2.9) from the compact stencils for the three-dimensional Laplacian is consistently ordered in the block form of (2.10).

Proof. Let $W=\{1,2, \ldots, N\}$ and $t=N$; set $S_{k}=\{k\}, k=1,2, \ldots, N$. We can verify that the sets $S_{1}, S_{2}, \ldots, S_{t}$ satisfy the conditions of Definition 2.3. Thus $A$ is consistently ordered in the block form.

LEMMA 3.2. Let $\left\{x_{j}\right\}_{j=0,1,2, \ldots, M+1}$ be a finite sequence, where $M$ is a given positive integer, and let $r$ be an unknown constant. Suppose $x_{j}$ and $r$ satisfy the boundary value problem of homogeneous linear difference equations

$$
x_{j+1}-2 r x_{j}+x_{j-1}=0,1 \leq j \leq M, \quad x_{0}=x_{M+1}=0 .
$$

Then the solutions of the problem are

$$
\begin{aligned}
& x_{j}(p)=2 i c \sin (j p h \pi), \quad 0 \leq j \leq M+1, p=1,2, \ldots, M, \\
& r(p)=\cos (p h \pi),
\end{aligned}
$$

where $i$ is the imaginary unit, $h=1 /(M+1)$ and $c$ is an arbitrary nonzero constant.

PROOF. Solving (3.1) without the boundary conditions, we have

$$
x_{j}=c_{1}\left(r+\sqrt{r^{2}-1}\right)^{j}+c_{2}\left(r-\sqrt{r^{2}-1}\right)^{j}, \quad 0 \leq j \leq M+1,
$$

where $c_{1}$ and $c_{2}$ are two arbitrary constants. On entering the boundary values into (3.2), we have

$$
c_{1}+c_{2}=0 \quad \text { and } \quad c_{1}\left(r+\sqrt{r^{2}-1}\right)^{M+1}+c_{2}\left(r-\sqrt{r^{2}-1}\right)^{M+1}=0 .
$$

Hence, we have

$$
\left(\frac{r+\sqrt{r^{2}-1}}{r-\sqrt{r^{2}-1}}\right)^{M+1}=1 \quad \text { or } \quad\left(r \pm \sqrt{r^{2}-1}\right)^{ \pm 2(M+1)}=1 .
$$

Notice that for $h=1 /(M+1)$ we obtain

$$
r(p) \pm \sqrt{r(p)-1}=\exp ( \pm i p \pi /(M+1))=\exp ( \pm i p h \pi), \quad p=1,2, \ldots, M
$$

In fact, (3.3) contains two equations. Adding them together gives rise to

$$
r(p)=\frac{1}{2}\left(e^{i p h \pi}-e^{-i p h \pi}\right)=\cos (p h \pi), \quad p=1,2, \ldots, M .
$$

Substituting (3.4) into (3.2) gives

$$
x_{j}(p)=c_{1}\left(e^{i j p h \pi}-e^{-i j p h \pi}\right)=2 i c \sin (j p h \pi), \quad 0 \leq j \leq M+1,
$$

and Lemma 3.2 is proved. 
THEOREM 3.3. Consider the linear system (2.9) yielded by the compact stencils for the three-dimensional Laplacian. If $B_{J}$ is the iteration matrix of the block-Jacobi method and $\rho\left(B_{J}\right)$ is its spectral radius, then we have

$$
\rho\left(B_{J}\right)=\frac{\left[\alpha+4 \cos (h \pi) \beta+4 \cos ^{2}(h \pi) \gamma\right] \cos (h \pi)}{[3-2 \cos (h \pi)] \alpha+\left[6-2 \cos ^{2}(h \pi)\right] \beta+4 \gamma} .
$$

PROOF. Let $B_{J}$ denote the block-Jacobi iteration matrix, $\lambda$ be an arbitrary eigenvalue of $B_{J}$ and $v$ be the corresponding eigenvector. Therefore $B_{J} v=\lambda v$, which leads to

$$
\begin{aligned}
& {\left[d v_{j, k, m}-\alpha\left(\sum_{j_{1}= \pm 1} v_{j+j_{1}, k, m}+\sum_{k_{1}= \pm 1} v_{j, k+k_{1}, m}\right)\right.} \\
& \left.\quad-\beta\left(\sum_{\substack{j_{1}= \pm 1 \\
k_{1}= \pm 1}} v_{j+j_{1}, k+k_{1}, m}\right)\right] \lambda=\alpha \sum_{m_{1}= \pm 1} v_{j, k, m+m_{1}} \\
& \quad+\beta \sum_{m_{1}= \pm 1}\left(\sum_{j_{1}= \pm 1} v_{j+j_{1}, k, m+m_{1}}+\sum_{k_{1}= \pm 1} v_{j, k+k_{1}, m+m_{1}}\right) \\
& \quad+\gamma \sum_{j_{1}= \pm 1} \sum_{k_{1}= \pm 1} \sum_{m_{1}= \pm 1} v_{j+j_{1}, k+k_{1}, m+m_{1}}, \\
& v_{0, k, m}=v_{N+1, k, m}=v_{j, 0, m}=v_{j, N+1, m}=v_{j, k, 0}=v_{j, k, N+1}=0 \quad 1 \leq j, k, m \leq N .
\end{aligned}
$$

System (3.5) is a boundary value problem of homogeneous linear difference equations. In order to determine $\lambda$ and $v$, we assume $v_{j, k, m}=x_{j} y_{k} z_{m}$ is a solution. Substitution of this term into (3.5) gives for $1 \leq j, k, m \leq N$

$$
\begin{aligned}
& {\left[d x_{j} y_{k} z_{m}-\alpha\left(\sum_{j_{1}= \pm 1} x_{j+j_{1}} y_{k} z_{m}+\sum_{k_{1}= \pm 1} x_{j} y_{k+k_{1}} z_{m}\right)\right.} \\
& \left.-\beta \sum_{\substack{j_{1}= \pm 1 \\
k_{1}= \pm 1}} x_{j+j_{1}} y_{k+k_{1}} z_{m}\right] \lambda=\alpha \sum_{m_{1}= \pm 1} x_{j} y_{k} z_{m+m_{1}} \\
& +\beta \sum_{m_{1}= \pm 1}\left(\sum_{j_{1}= \pm 1} x_{j+j_{1}} y_{k} z_{m+m_{1}}+\sum_{k_{1}= \pm 1} x_{j} y_{k+k_{1}} z_{m+m_{1}}\right) \\
& +\gamma \sum_{j_{1}= \pm 1} \sum_{k_{1}= \pm 1} \sum_{m_{1}= \pm 1} x_{j+j_{1}} y_{k+k_{1}} z_{m+m_{1}},
\end{aligned}
$$


and

$$
x_{0}=x_{N+1}=0, \quad y_{0}=y_{N+1}=0, \quad z_{0}=z_{N+1}=0 .
$$

This can be simplified to

$$
\frac{d x_{j} y_{k}-\alpha a-\beta b}{\alpha x_{j} y_{k}+\beta a+\gamma b} \lambda=\frac{z_{m+1}+z_{m-1}}{z_{m}} \quad 1 \leq j, k, m \leq N,
$$

where

$$
a=\sum_{j_{1}= \pm 1} x_{j+j_{1}} y_{k}+\sum_{k_{1}= \pm 1} x_{j} y_{k+k_{1}}, \quad b=\sum_{j_{1}= \pm 1} \sum_{k_{1}= \pm 1} x_{j+j_{1}} y_{k+k_{1}} .
$$

Since the left-hand side of (3.6) depends on $j$ and $k$ while the right-hand side depends on $m$, the two sides are independent. This implies that both sides must be a constant. Let this constant be $2 r$. Then we have

$$
\begin{gathered}
z_{m+1}-2 r z_{m}+z_{m-1}=0 \quad 1 \leq m \leq N, \quad z_{0}=z_{N+1}=0, \\
\left(\frac{\lambda}{2 r} d-\alpha\right) x_{j} y_{k}=\left(\frac{\lambda}{2 r} \alpha+\beta\right) a+\left(\frac{\lambda}{2 r} \beta+\gamma\right) b .
\end{gathered}
$$

Equation (3.8) can be written as

$$
\begin{gathered}
\frac{\left[(\lambda d-2 r \alpha) /(\lambda \beta+2 r \gamma)+(\lambda \alpha+2 r \beta)^{2} /(\lambda \beta+2 r \gamma)^{2}\right] x_{j}}{x_{j+1}+(\lambda \alpha+2 r \beta) /(\lambda \beta+2 r \gamma) x_{j}+x_{j-1}} \\
=\frac{y_{k+1}+(\lambda \alpha+2 r \beta) /(\lambda \beta+2 r \gamma) y_{k}+y_{k-1}}{y_{k}} .
\end{gathered}
$$

Repeat the similar process of separating variables and let both sides of (3.9) equal a constant $2 s$. We obtain

$$
\begin{gathered}
y_{k+1}-2\left[s-\frac{\lambda \alpha+2 r \beta}{2(\lambda \beta+2 r \gamma)}\right] y_{k}+y_{k-1}=0 \quad 1 \leq k \leq N, \\
y_{0}=y_{N+1}=0,
\end{gathered}
$$

and

$$
\begin{gathered}
x_{j+1}-2\left[\frac{(\lambda d-2 r \alpha)(\lambda \beta+2 r \gamma)+(\lambda \alpha+2 r \beta)^{2}}{4 s(\lambda \beta+2 r \gamma)^{2}}\right. \\
\left.-\frac{\lambda \alpha+2 r \beta}{2(\lambda \beta+2 r \gamma)}\right] x_{j}+x_{j-1}=0 \quad 1 \leq k \leq N, \\
x_{0}=x_{N+1}=0 .
\end{gathered}
$$

By Lemma 3.2, solving (3.7), (3.10) and (3.11) gives

$$
r(p)=\cos (p h \pi), \quad s(q)-\frac{\lambda \alpha+2 r(p) \beta}{2[\lambda \beta+2 r(p) \gamma]}=\cos (q h \pi)
$$


TABLE 1. Formulae for $\omega_{b}$ for seven-point, 15-point, 19-point and 27-point stencils.

\begin{tabular}{|c|c|}
\hline Stencil & $\omega_{b}$ \\
\hline \multirow{2}{*}{ 7-point } & 2 \\
\hline & $1+\sqrt{1-[\cos (h \pi) /(3-2 \cos (h \pi))]^{2}}$ \\
\hline \multirow{2}{*}{15 -point } & 2 \\
\hline & $1+\sqrt{1-\left[\left(2+\cos ^{2}(h \pi)\right) /(7-4 \cos (h \pi)) \cos (h \pi)\right]^{2}}$ \\
\hline \multirow{2}{*}{ 19-point } & 2 \\
\hline & $1+\sqrt{1-\left\{([1+2 \cos (h \pi)] \cos (h \pi)) /\left(7-[1+\cos (h \pi)]^{2}\right)\right\}^{2}}$ \\
\hline \multirow{2}{*}{ 27-point } & 2 \\
\hline & $1+\sqrt{1-\left\{\left([2+\cos (h \pi)]^{2} \cos (h \pi)\right) /\left(27-2[2+\cos (h \pi)]^{2}\right)\right\}^{2}}$ \\
\hline
\end{tabular}

and

$$
\begin{aligned}
& \frac{(\lambda d-2 r(p) \alpha) /(\lambda \beta+2 r(p) \gamma)+\left([\lambda \alpha+2 r(p) \beta]^{2}\right) /\left([\lambda \beta+2 r(p) \gamma]^{2}\right)}{4 s(q)} \\
& -\frac{\lambda \alpha+2 r(p) \beta}{2[\lambda \beta+2 r(p) \gamma]}=\cos (\operatorname{lh} \pi),
\end{aligned}
$$

where $p, q, l=1,2, \ldots, N$.

Upon solution of the above equations, the $N^{3}$ eigenvalues of $B_{J}$ can be expressed as

$$
\lambda(p, q, l)=\frac{\{\alpha+2[\cos (q h \pi)+\cos (h h \pi)] \beta+4 \cos (q h \pi) \cos (l h \pi) \gamma\} \cos (p h \pi)}{[3-\cos (q h \pi)-\cos (l h \pi)] \alpha+[6-2 \cos (q h \pi) \cos (l h \pi)] \beta+4 \gamma} .
$$

Consequently, the spectral radius of $B_{J}$ can be given as

$$
\rho\left(B_{J}\right)=\max _{p, q, l}|\lambda(p, q, l)|=\frac{\left[\alpha+4 \cos (h \pi) \beta+4 \cos ^{2}(h \pi) \gamma\right] \cos (h \pi)}{[3-2 \cos (h \pi)] \alpha+\left[6-2 \cos ^{2}(h \pi)\right] \beta+4 \gamma} .
$$

Hence, Theorem 3.3 is proved.

By Theorems 2.5 and 3.3 and Lemma 3.1, the following corollary is obtained.

COROLLARY 3.4. The optimum relaxation parameter of block-SOR iteration for the compact stencil for three-dimensional Laplacian, $\omega_{b}$, can be given as

$$
\omega_{b}=2 /\left(1+\sqrt{1-\left\{\frac{\left[\alpha+4 \cos (h \pi) \beta+4 \cos ^{2}(h \pi) \gamma\right] \cos (h \pi)}{[3-2 \cos (h \pi)] \alpha+\left[6-2 \cos ^{2}(h \pi)\right] \beta+4 \gamma}\right\}^{2}}\right) .
$$


(a) Point-SOR

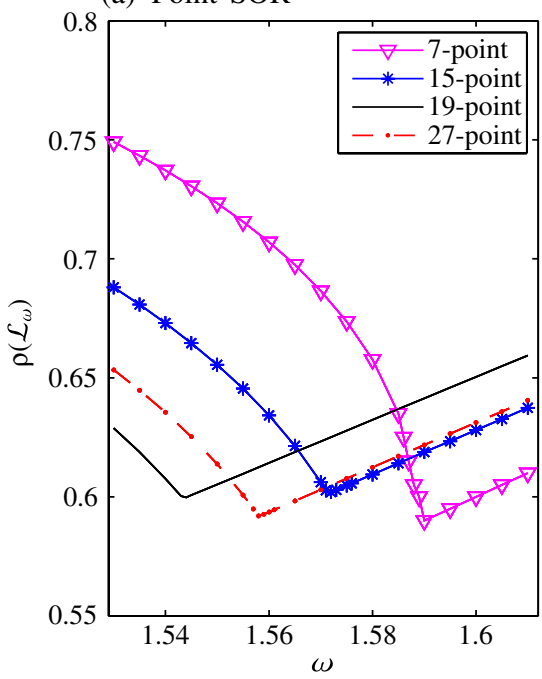

(b) Block-SOR

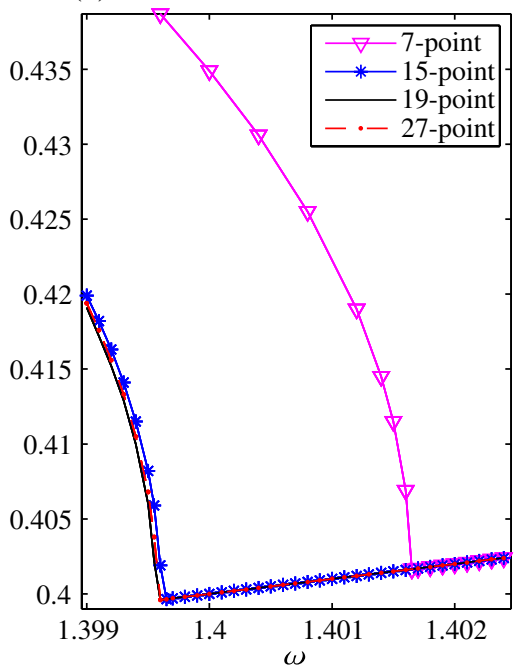

FIGURE 3. $\rho\left(\mathcal{L}_{\omega}\right)$ versus $\omega$ for the four compact stencils (seven-point, 15-point, 19-point and 27-point) with $h=1 / 12$.

By (2.5)-(2.8) and (3.12), the optimum relaxation parameters of block-SOR iteration for seven-point, 15-point, 19-point and 27-point stencils for the threedimensional Laplacian can be determined (see Table 1).

Figure 3 shows two graphs of $\rho\left(\mathcal{L}_{\omega}\right)$ as a function of $\omega$ for the case $h=1 / 12$ using numerical simulation. Figure 3(a) shows the results for point-SOR and Figure 3(b) shows those for block-SOR.

From Figure 3 we can see the following.

(1) With optimum relaxation parameters, $\rho\left(\mathcal{L}_{\omega}\right)$ of block-SOR (b) is much smaller than that of point-SOR (a).

(2) By Table 1 for $h=1 / 12$, the optimum relaxation parameters of block-SOR for the four compact stencils are: seven-point stencil $\omega_{b}^{*} \doteq 1.401641 ; 15$-point stencil $\omega_{b}^{*} \doteq 1.399608 ; 19$-point stencil $\omega_{b}^{*} \doteq 1.399560 ; 27$-point stencil $\omega_{b}^{*} \doteq$ 1.399 576. From Figure 3(b), we can see the numerical results agree well with the theoretical results.

(3) In block-SOR, using their optimum relaxation parameters, the 15-point, 19-point and 27-point stencils hold nearly the same values for $\rho\left(\mathcal{L}_{\omega}\right)$, which are a little smaller than the seven-point stencil.

\section{Conclusions}

The block-SOR iteration method with natural row-wise ordering has been analysed for the compact stencils for the three-dimensional Laplacian. The technique of separation of variables was used to determine the optimum relaxation parameters of 
the methods. Analyses show that block-SOR iteration has advantages over pointSOR iteration when solving a three-dimensional Laplacian problem. According to the numerical simulations shown in Figure 3, we see that $\rho\left(\mathcal{L}_{\omega}\right)$ is very sensitive to $\omega$ around $\omega_{b}$ for block-SOR iteration. Therefore, accuracy is critical in applications. Our results provide a theoretical solution for determining optimal $\omega_{b}$.

For the two-dimensional Laplacian, we can deduce the optimum relaxation parameters through a similar use of separation of variables. Therefore, the optimum relaxation parameters for block-SOR iteration with natural row-wise ordering for the five-point and nine-point Laplacian can be given directly.

\section{Acknowledgement}

This work is supported in part by the NSFC (No. 60771042), 863 Program (No. 2007AA12Z159) and the program for new century excellent talents in University of China.

\section{References}

[1] L. M. Adams, R. J. LeVeque and D. M. Young, "Analysis of the SOR iteration for the 9-point Laplacian", SIAM J. Numer. Anal. 25 (1988) 1156-1180.

[2] D. Xie and L. Adams, "New parallel SOR method by domain partitioning", SIAM J. Sci. Comput. 20 (1999) 2261-2281.

[3] A. Hadjidimos, "Successive overrelaxation (SOR) and related methods", J. Comput. Appl. Math. 123 (2000) 177-199.

[4] R. J. Leveque and L. N. Trefethen, "Fourier analysis of the SOR iteration", IMA J. Numer. Anal. 8 (1988) 273-279.

[5] A. I. Van de Vooren and A. C. Vliegenthart, "On the 9-point difference formula for Laplace's equation", J. Engrg. Math. 1 (1967) 187-202.

[6] D. M. Young, "Iterative methods for solving partial difference equations of elliptic type", Doctoral Thesis, Harvard University, Mathematical Department, Cambridge, Massachusetts, 1950.

[7] D. M. Young, "Iterative methods for solving partial difference equations of elliptic types", Trans. Amer. Math. Soc. 76 (1954) 92-111.

[8] D. M. Young, Iterative solution of large linear systems (Academic Press, New York-London, 1971). 